\title{
Writing Effective Paragraphs
}

\author{
Kristin Messuri PhD
}

\begin{abstract}
Taking a methodical approach to constructing paragraphs can improve clarity and organization in medical writing. This article describes a typical model for paragraph structure, explains the significance of coherence and cohesion, and recommends revision strategies.
\end{abstract}

Key words: writing, medical writing, medical manuscript, periodicals as topic

Almost all writers realize the need to group sentences into paragraphs, yet many do not approach this aspect of writing methodically. When asked how they determined paragraph structure, writers often reveal that they did not have a strategy, indicate that the paragraph divisions "just felt right," or explain that they began a new paragraph when the previous paragraph seemed too long. Some of these responses suggest a nascent understanding of paragraphing (organizing sentences into a paragraph). A paragraph indicates that the sentences it contains are related and somehow distinct from the other sentences in the text. Due to this function, paragraphs have been called "macropunctuation": they function as rhetorical signifiers that help an audience understand a text. ${ }^{1}$

Thinking critically about how paragraphing affects the delivery of a message can help authors to enhance the clarity and organization of their writing. Therefore, the remainder of this article will describe paragraph structure, coherence, and cohesion, as well as propose methods for revising paragraphs.

Corresponding author: Kristin Messuri, Ph.D. Contact Information: kristin.messuri@gmail.com DOI: 10.12746/swrccc 2016.0415.209

\section{Paragraph Structure}

Authors must structure their paragraphs effectively in order to develop their discussions and show the relationships between ideas. In general, in medical writing, a paragraph has three main components: a topic sentence, supporting sentences, and a concluding sentence. The topic sentence is usually the first sentence of the paragraph; it states the paragraph's central concept or argument. Often, a topic sentence introduces a new concept and/or transitions from the previous concept by showing the relationship between the two. Supporting sentences provide explanations, evidence, and other details related to the paragraph's main concept. Paragraphs typically end with concluding sentences, which provide the reader with a sense of closure. A concluding sentence may take many forms, including a brief summary of the paragraph's information or a statement about the implications of that information. However, ending paragraphs with transitions is usually not effective. Introducing a new concept in the final sentence of a paragraph will likely confuse readers. Instead, this transition should appear in the topic sentence of the next paragraph. Of course, this paragraph structure is not the only possibility; it is simply one common structure that is effective for most medical writing tasks. 
Paragraph divisions and length are determined by the concept developed in that paragraph. Writers may create divisions between paragraphs for a number of reasons, including to address a new concept, to compare or contrast information, to introduce a counter-argument, or to give readers a chance to process information before moving on. Although there are no strict guidelines for paragraph length, each paragraph should be long enough to fully develop a concept but not so long that readers become tired, bored, or confused. Writing paragraphs of an effective length is, then, a matter of being considerate to the audience. ${ }^{2}$

\section{Paragraph Coherence and Cohesion}

Both coherence and cohesion are important qualities of effective paragraphs and texts. These terms are sometimes used interchangeably, but for the purposes of this article, coherence refers to the clarity and comprehensibility of a text or a unit of a text (such as a paragraph), whereas cohesion refers to the sentence-level structures and words that foster this sense of unity. Even when the overall organization is predetermined, as in the common "introduction, methodology, results, and discussion" (IMRAD) model, ${ }^{3}$ writers develop coherence by clarifying the relationships between sections and individual paragraphs as well as clearly communicating the meaning of their ideas. In coherent medical writing, paragraphs are ordered logically; each paragraph has one central, well-developed concept; relationships between ideas are adequately explained; and the language is appropriate for the audience.

Cohesion fosters coherence through the inclusion of transitions between ideas, as well as effective sentence structure and word choice. Cohesion is sometimes called "flow"; it can be described as the "glue" holding the paragraph together. In a cohesive paragraph, signal words or transitions will connect each sentence to the sentences before and after it. Moreover, key terms will be repeated and used consistently. Note that it is possible for a paragraph to be cohesive, but not coherent. Ideas may be connected, but they may use language that is far too technical for the audience, causing confusion. Or, each sentence may have effective language, explanations, and tran- sitions but may fail to adequately develop a central idea, rendering the paragraph incomprehensible.

\section{Conclusion: Revising Paragraphs}

As with most aspects of writing, paragraphs are best revised through a careful consideration of audience. With a specific audience in mind, a writer should evaluate how the content of each paragraph develops the central argument of the paper. Try to view the text from the perspective of a journal reader, a conference attendee, and so forth. Does each paragraph provide new information that serves a specific purpose in the text? Or, do some paragraphs either provide extraneous information or digress from the text's main focus, signs that revisions are necessary?

A writer should ensure that (1) each paragraph has one central idea and (2) those paragraphs appear in a logical order in keeping with the organizational conventions of the genre (such as the IMRAD model). One method discussed in a past article in this series is preparing a reverse outline by listing the central idea of each paragraph. ${ }^{4}$ This process helps a writer to determine whether each paragraph does, indeed, contain only one central idea. If a paragraph contains multiple concepts, the writer may need to divide the paragraph or move or delete information. Once the outline is prepared, the writer can evaluate whether the paragraphs (represented by their main ideas) are in a logical order and whether they all support the main argument of the text. For example, are the paragraphs in the methods section divided logically? Do they build on one another to explain how the research was conducted?

Another useful method is writing down the text's thesis or central argument followed by the topic sentence of each paragraph. The result is a rough outline that should progress logically through the main ideas of the text. If the outline is difficult to understand, topic sentences may need to be rewritten or paragraphs may need to be moved, deleted, or otherwise revised.

Finally, the writer should evaluate each paragraph for coherence and cohesion by examining the 
connections between ideas, syntax, and linguistic choices.

Author Affiliations: Kristin Messuri is the Associate Director of the University Writing Center at Texas Tech University / Texas Tech University Health Sciences Center in Lubbock, TX

Submitted: 7/1/2016

Published electronically: 7/15/2016

Conflict of Interest Disclosures: None

\section{REFERENCES}

1. Reeves A, Leventhal P. Paragraphing (part 1 of 2). Med Writ 2012; 21 (4): 298-304.

2. Taylor RB. Medical writing: a guide for clinicians, educators, and researchers. 2nd ed. New York: Springer; 2012.

3. Sollaci LB, Pereira, MG. The introduction, methods, results, and discussion (IMRAD) structure: a fifty-year survey. J Med Libr Assoc 2004; 92 (3): 364-371.

4. Messuri K. Revision strategies. Southwest Respir Crit Care Chron 2016; 4 (14): 46-48. 